\title{
Evaluation of the effect of experimentally induced cartilage defect and intra-articular hyaluronan on synovial fluid biomarkers in intercarpal joints of horses
}

\author{
Tytti Maaria Niemelä ${ }^{*}$, Riitta-Mari Tulamo ${ }^{1}$, Jorge Uriel Carmona ${ }^{2}$ and Catalina López ${ }^{2}$
}

\begin{abstract}
Background: Inflammatory and degenerative activity inside the joint can be studied in vivo by analysis of synovial fluid biomarkers. In addition to pro-inflammatory mediators, several anabolic and anti-inflammatory substances are produced during the disease process. They counteract the catabolic effects of the pro-inflammatory cytokines and thus diminish the cartilage damage. The response of synovial fluid biomarkers after intra-articular hyaluronan injection, alone or in combination with other substances, has been examined only in a few equine studies. The effects of hyaluronan on some pro-inflammatory mediators, such as prostaglandin $E_{2}$, have been documented but especially the effects on synovial fluid anti-inflammatory mediators are less studied. In animal models hyaluronan has been demonstrated to reduce pain via protecting nociceptive nerve endings and by blocking pain receptor channels. However, the results obtained for pain-relief of human osteoarthritis are contradictory. The aim of the study was to measure the synovial fluid IL-1 ra, PDGF-BB, TGF- $\beta_{1}$ and TNF-a concentrations before and after surgically induced cartilage defect, and following intra-articular hyaluronan injection in horses. Eight Standardbred horses underwent bilateral arthroscopic surgeries of their intercarpal joints under general anaesthesia, and cartilage defect was created on the dorsal edge of the third carpal bone of one randomly selected intercarpal joint of each horse. Five days post-surgery, one randomly selected intercarpal joint was injected intra-articular with $3 \mathrm{~mL} H \mathrm{HA}(20 \mathrm{mg} / \mathrm{mL})$.

Results: Operation type had no significant effect on the synovial fluid IL-1 ra, PDGF-BB, TGF- $\beta_{1}$ and TNF-a concentrations but compared with baseline, synovial fluid IL-1 ra and TNF-a concentrations increased. Intra-articular hyaluronan had no significant effect on the biomarker concentrations but a trend of mild improvement in the clinical signs of intra-articular inflammation was seen.

Conclusions: Creation of the cartilage defect and sham-operation lead to an increase of synovial fluid IL-1 ra and TNF-a concentrations but changes in concentrations of anabolic growth factors TGF- $\beta_{1}$ and PDGF-BB could not be documented 5 days after the arthroscopy. Intra-articular hyaluronan was well tolerated. Further research is needed to document possible treatment effects of intra-articular hyaluronan on the synovial fluid biomarkers of inflammation and cartilage metabolism.
\end{abstract}

Keywords: Experimental study, Cartilage defect, Hyaluronan, Interleukin-1 receptor antagonist, Non-animal stabilized hyaluronic acid (NASHA), Platelet-derived growth factor BB, Synovitis, Transforming growth factor $\beta_{1}$, Tumour necrosis factor a

\footnotetext{
*Correspondence: tytti.niemela@helsinki.fi

${ }^{1}$ Department of Equine and Small Animal Medicine, Faculty of Veterinary

Medicine, University of Helsinki, P.O. Box 57, 00014 Helsinki, Finland

Full list of author information is available at the end of the article
} 


\section{Background}

Research on equine joint disease has been focused on finding tools for early diagnosis and monitoring the treatments and progression of the joint disease. Inflammatory and degenerative activity in the joint can be studied in vivo by analysis of synovial fluid (SF) biomarkers, such as pro-inflammatory cytokines in experimental setting [1-5], or in naturally occurring joint disease [6-8]. In addition to pro-inflammatory mediators, several anabolic and anti-inflammatory mediators are produced during the inflammatory process. They counteract the catabolic effects of the pro-inflammatory cytokines and diminish the cartilage damage. Moreover, the anti-inflammatory effect can produce temporal relief of the clinical symptoms of the articular disease [9].

Equine arthritis is commonly treated with intra-articular (IA) corticosteroids and hyaluronan (HA) [10]. However, the response of SF biomarkers after IA HA injection, alone or in combination with other substances, has been examined only in a few equine studies [3, 4 , 11]. In human medicine, clinical efficacy of HA has been widely studied [12]. In addition, the effect of IA HA on some SF pro-inflammatory biomarkers, such as prostaglandin $\mathrm{E}_{2}$, has been explored both in human and equine studies [3, 4, 13-15]. However, research on the effect of HA on anti-inflammatory mediators has been infrequently conducted. Despite the observed effects of HA on pro-inflammatory mediators, results in pain-relief in human osteoarthritis (OA) are contradictory [12]. In animal models HA has been demonstrated to reduce pain via protecting nociceptive nerve endings [16] and by blocking pain receptor channels [17].

The aim of the study was to measure SF concentrations of the anti-inflammatory mediator interleukin-1 receptor antagonist (IL-1ra), the anabolic growth factors plateletderived growth factor BB (PDGF-BB) and transforming growth factor beta-1 (TGF- $\left.\beta_{1}\right)$ and the pro-inflammatory cytokine tumour necrosis factor alpha (TNF- $\alpha$ ) before and after surgically induced cartilage defect (CD) in healthy horses. Secondly, we wanted to explore if the concentrations of the selected biomarkers changed following the IA HA (non-animal stabilized hyaluronic acid, NASHA $^{1}$ ) injection. The hypothesis was that both concentrations of anti-inflammatory cytokines and concentration of TNF- $\alpha$ will increase in SF after the induction of $\mathrm{CD}$; and that in the HA-injected joints the pro-inflammatory TNF- $\alpha$ is decreased and the concentrations of antiinflammatory mediators are increased compared with the joints without the HA medication.

${ }^{1}$ Durolane, Bioventus LLC, Durham, NC, USA.

\section{Methods}

The study protocol was approved by the National Animal Experimental Board in Finland. Eight Standardbred horses (four mares, one stallion and three geldings) free of lameness were used. Before recruiting the horses, combination of interventions was randomly picked for each right intercarpal joint. As a result, each joint was selected for one of the following; cartilage defect (CD) with $\mathrm{HA}, \mathrm{CD}$ without injection, sham-operation (SO) with $\mathrm{HA}$ or $\mathrm{SO}$ without injection. Interventions for each of the contralateral intercarpal joints were determined by these randomly picked combinations; i.e. if $\mathrm{CD}$ with $\mathrm{HA}$ was selected for the right side, left side was for SO without injection.

The median age of horses was 7 years and range 4-24 years. Examinations, grading of measured variables and surgical procedures were carried by the principal veterinarian (TMN). Prior to inclusion, horses were subjected to a complete lameness examination. A standardized American Association of Equine Practitioners' (AAEP) scale of 0-5 [18] was used to grade lameness. Effusion of the affected joint was recorded on a scale of $0-4(0=$ no effusion, $1=$ mild, $2=$ moderate, $3=$ severe effusion, $4=$ severe swelling of the joint region) [19]. A flexion test of the affected and the contralateral limb was performed, and lameness was recorded on a scale of $0-4(0=$ no increase, $1=$ slight increase, $2=$ moderate increase, $3=$ considerable increase compared with the baseline lameness, $4=$ non-weight-bearing lameness) [3]. Pain score for maximal flexion of the carpus was also determined and recorded on a scale of $0-3(0=$ no pain on flexion, $1=$ mild pain, i.e. the horse shows some reaction, such as moving the limb, $2=$ moderate pain, i.e. the horse retracts the limb repeatedly during the $1 \mathrm{~min}$ flexion period, $3=$ severe pain, i.e. the flexion test cannot be properly performed). In addition, five radiographic views (dorsopalmar, dorsolateral-palmaromedial, dorsomedialpalmarolateral, flexed lateromedial and flexed dorsoproximal-dorsodistal) of the carpal joints were assessed.

Before the surgical procedure, $5 \mathrm{~mL}$ of the SF of both intercarpal joints of each horse were aspirated into a sterile $5 \mathrm{~mL}$ syringe for the biomarker measurements. The SF sample was immediately divided between a plain $4 \mathrm{~mL}$ tube on ice and into an etylenediaminetetraacetic acid (EDTA) tube. White blood cell (WBC) count and total protein (TP) concentration measurements were done from the fresh sample in the EDTA tube. Within $1 \mathrm{~h}$ of collection, the plain sample was centrifuged at $4000 \mathrm{rpm}$ for $10 \mathrm{~min}$ in $4{ }^{\circ} \mathrm{C}$, aliquoted and stored at $-80^{\circ} \mathrm{C}$.

The horses underwent bilateral arthroscopic surgeries of their intercarpal joints under general anaesthesia. No pre-existing IA abnormalities were detected during the arthroscopy in any of the joints. Cartilage defect 
was created on the dorsal edge of the third carpal bone of one randomly selected intercarpal joint of each horse. The lesion was generated using a $5.0 \mathrm{~mm} \times 13 \mathrm{~cm}$ arthroscopic burr. ${ }^{2}$ After the procedure, the debris was left in the joint, inducing synovitis and articular inflammation. Synovial membrane and joint capsule (approximately $3 \mathrm{~mm} \times 3 \mathrm{~mm}$ ) were harvested with a scalpel from the dorsal region of the joints for a study to be reported elsewhere. The sham-operated contralateral joints (SO) served as controls and were similarly evaluated by arthroscopic examination, synovial membranes and joint capsules were harvested but the cartilage was left intact. The arthroscopic portals were closed, forelimbs were bandaged, and horses were allowed to recover from the anaesthesia and surgery. The horses were housed in stall boxes. The status of each horse was monitored three times daily, including comfort, lameness at walk, body temperature, heart rate and respiratory rate.

Five days post-surgery, the lameness examination was repeated, new SF samples from both intercarpal joints were harvested and one randomly selected intercarpal joint was injected IA with $3 \mathrm{~mL}$ HA $(20 \mathrm{mg} / \mathrm{mL})$. Nine days after the IA HA injection (i.e. 2 weeks after the surgical arthroscopic procedure) the third lameness evaluation was done, and the third SF sampling, and the second synovial soft tissue sampling of both intercarpal joints were performed under general anesthesia, after which horses were euthanized on the operating table.

\section{Laboratory analyses}

Synovial fluid samples obtained on day 0 , day 5 and day 14 were used to analyze for the concentration of IL-1ra, PDGF-BB, TGF- $\beta_{1}$ and TNF- $\alpha$. All markers were assayed using commercial ELISA development kits ${ }^{3},{ }^{4},{ }^{5},{ }^{6}$ from $R \& D$ Systems. Samples were analysed in triplicate. TGF$\beta_{1}$ (see footnote 3) and PDGF-BB (see footnote 4) were determined using human antibodies. IL-1ra (see footnote 5 ) and TNF- $\alpha$ (see footnote 6 ) were assayed with equinespecific antibodies. The standards provided for each ELISA kit were used in preparing each standard curve according to the manufacturers' instructions. Readings were performed at $450 \mathrm{~nm}$. The inter-, and intra-assay coefficient of variation was $<6 \%$ for each ELISA.

\footnotetext{
${ }^{2}$ Dyonics Arthroscopic Surgery Blade, Smith \& Nephew, Andover, MA, USA.

${ }^{3}$ Human TGF- $\beta 1$ DuoSet, DY240E, R\&D Systems, Minneapolis, MN, USA. ${ }^{4}$ Human PDGF-BB DuoSet, DY220, R\&D Systems, Minneapolis, MN, USA.

${ }^{5}$ Equine IL-1ra/IL1F3 DuoSet, DY1814, R\&D Systems, Minneapolis, MN, USA.

${ }^{6}$ Equine TNF-alphaDuoSet, DY1814, R\&D Systems, Minneapolis, MN, USA.
}

\section{Statistical methods}

IL-1ra, PDGF-BB, TGF- $\beta_{1}$, TNF- $\alpha$, WBC count and TP concentration were analysed with analysis of covariance models (ANCOVA). The study design had 3 time-points and 2 different interventions (operation, treatment). The two effects were analysed separately due to small sample size. The change in biomarker and TP concentrations and WBC count from pre-operation to pre-treatment was examined in one analysis and the change from pretreatment to end of follow-up in another analysis. In both analyses, the change in concentration was used as the response, operation type or treatment as the fixed effect and the corresponding baseline measurement as a covariate.

As there were still some doubts about the normality of distributions after transformation, the changes in biomarker and TP concentrations and WBC count were analysed also using the Wilcoxon signed rank test. The Wilcoxon test was conducted also for all 16 limbs (effect of operation regardless of type). A P-value $<0.05$ was accepted as statistically significant for all tests.

\section{Results \\ Clinical outcomes}

Five days after arthroscopy, all the horses showed signs of lameness and increased score in the flexion test of the $\mathrm{CD}$-limb and effusion of the affected joint. The mean lameness score of the CD-limbs was 2.5, the mean flexion test score was 2.9 and the mean effusion score was 2.1, respectively. Two horses showed bilateral lameness, i.e. had a mildly lame SO-limb. In the SO-group, the mean scores of the flexion test and effusion were 1 and 1.8, respectively. No pain was elicited in the operated joint in maximal flexion in either of the groups (CD, $\mathrm{SO})$.

Following trends can be seen in Fig. 1. After IA HA injection, the mean lameness score of the $C D$ group with IA HA (CD+HA) decreased more compared to the group without HA-injection ( $\mathrm{CD}-\mathrm{HA})$. Mild improvement in the flexion test score and more pronounced improvement in the effusion score were observed in the $\mathrm{CD}$ affected limbs of horses after IA HA (CD + HA) compared to the limbs without HA injections (CD-HA). Compared with $\mathrm{CD}$ groups, changes in the clinical scores and differences between the $\mathrm{SO}+\mathrm{HA}$ and $\mathrm{SO}-\mathrm{HA}$ groups were not consistent.

\section{Biomarker outcomes}

In the ANCOVA models, no statistically significant differences in the Type III tests of fixed effects were documented: no effects were documented by the type of operation (CD vs. SO) or treatment (HA vs. no HA) to the concentrations measured in SF biomarkers. However, differences between the time points were revealed: the 


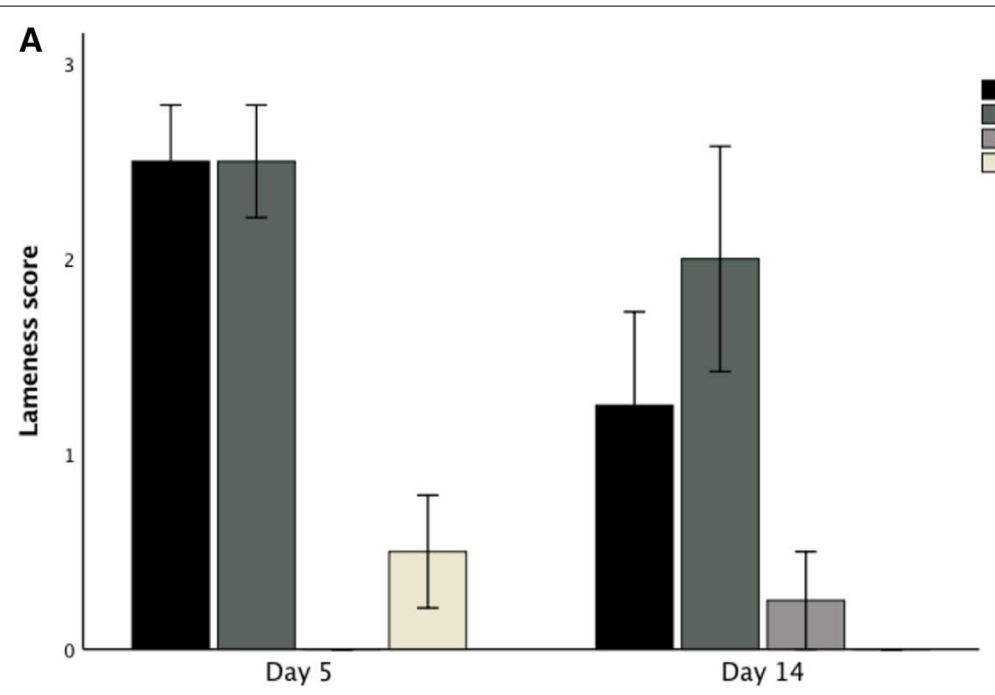

D $\mathrm{CD}+\mathrm{HA}$

CD-HA

SO+HA

SO-HA

B

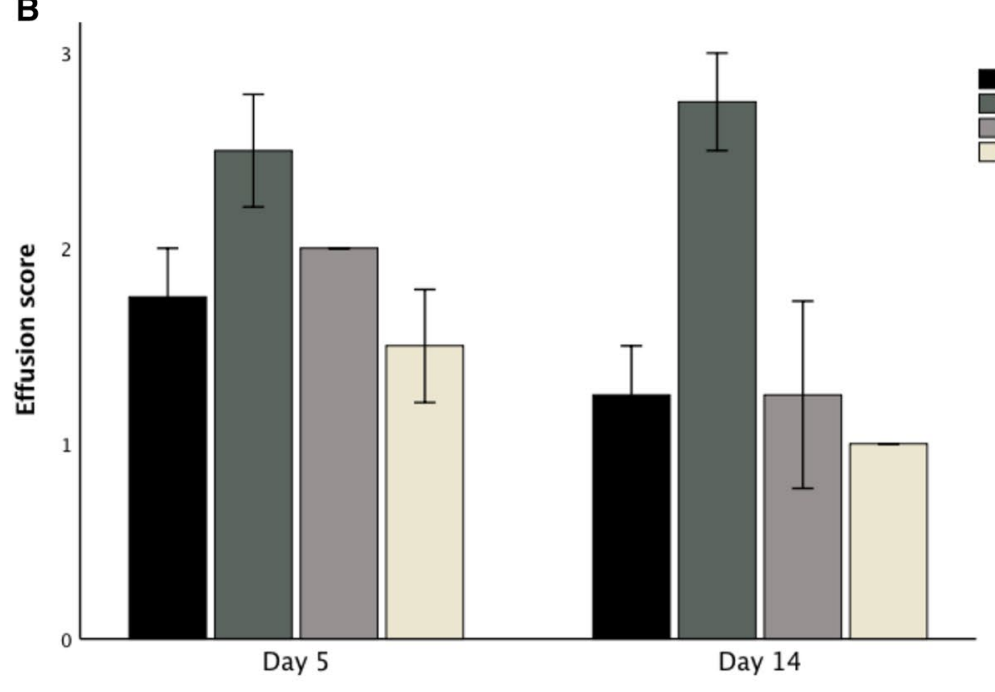

C

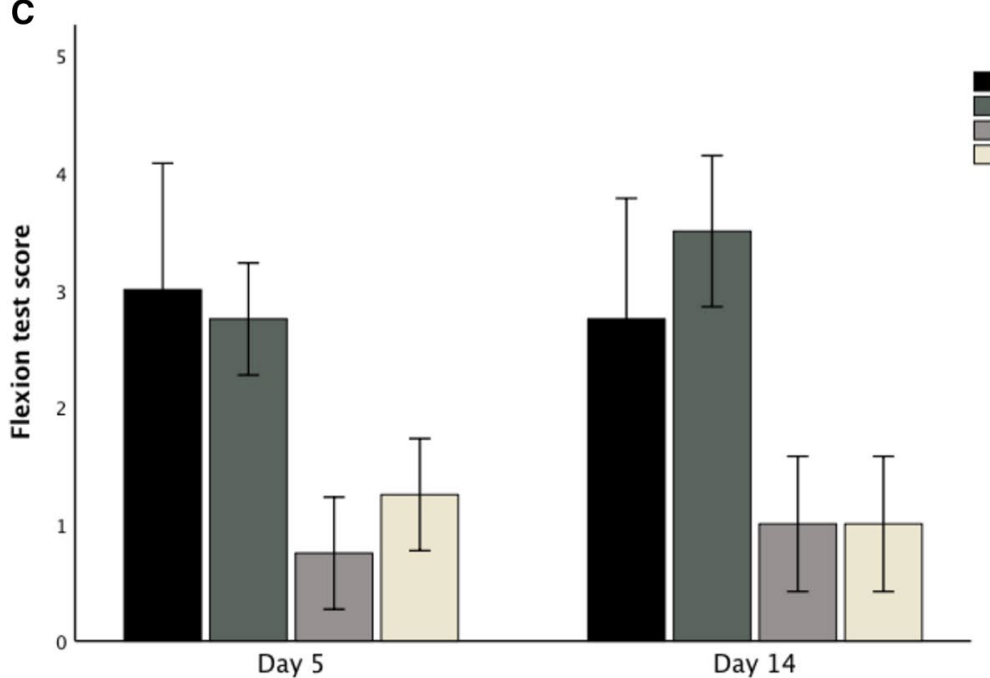

Fig. 1 Mean $( \pm S E M)$ clinical outcome measures of treatment groups post operation (day 5) and post treatment (day 14). $C D+H A$ cartilage defect joints with hyaluronan injection, $C D-H A$ cartilage defect joints without hyaluronan injection, $S O+H A$ sham-operated joints with hyaluronan injection, $\mathrm{SO}-\mathrm{HA}$ sham-operated joints without hyaluronan injection 
change from pre-operation to pre-treatment values were significant for $\mathrm{IL}-1 \mathrm{ra}(\mathrm{P}=0.0344$ in the $\mathrm{CD}$ group and $\mathrm{P}=0.0103$ in the $\mathrm{SO}$ group), WBC count $(\mathrm{P}=0.011$ in the $\mathrm{CD}$ group and $\mathrm{P}=0.002$ in $\mathrm{SO}$ group $)$ and $\mathrm{TP}(\mathrm{P}=0.0002$ in the $\mathrm{CD}$ group and $\mathrm{P}=0.002$ in the $\mathrm{SO}$ group). HA had no significant effect on the biomarker concentrations within the groups.

In the Wilcoxon signed rank tests similar results were seen. The SF IL-1ra concentration was not significantly different for the operation types (CD vs. SO) but regarding all limbs the SF IL-1ra concentration increased significantly after arthroscopy $(P=0.0039)$. Also, the increase in SF TNF- $\alpha$ concentration was significant $(P=0.0386)$ regarding all limbs. Induction of $\mathrm{CD}$ and $\mathrm{SO}$ both caused a significant increase within both groups in WBC count $(\mathrm{P}<0.001$ in both groups) and $\mathrm{TP}$ concentration $(\mathrm{P}<0.001$ in both groups).

\section{Discussion}

In the present study, the concentrations of the measured biomarkers in intact equine joints are mainly equivalent compared with the results of a previous report [20]. To the best of our knowledge, IL-1ra, PDGF-BB, TGF- $\beta_{1}$ measured in the present study have been evaluated separately only in a few studies on SF of the equine joint [2, 21-23]. A purely catabolic cytokine TNF- $\alpha$ has been studied in greater detail [5, 20, 21, 23-28]. Most of the biomarkers have been documented in vitro in equine cartilage as well as in the synovial membrane after a challenge, usually lipopolysaccharide (LPS) [20, 29-31].

IL-1ra has been shown to increase after an acute intraarticular fracture in humans. However, differences in SF inflammatory cytokine concentrations between high and low-energy injuries have not been detected [32]. Although not directly comparable with intra-articular fractures, in the present study no differences were detected between CD joints and SO joints. However, the arthroscopy itself caused trauma to the synovial soft tissues and may have caused the increase of IL-1ra concentration.

Blocking the IL-1 $\beta$ receptor by IL-1ra has potentially a wide positive effect on inhibiting deleterious events in the joint. The binding of IL- $1 \beta$ to a receptor results in activation of several transcription factors and expression of hundreds of genes leading to the synthesis of other cytokines, chemokines, adhesion molecules, inflammatory mediators and enzymes [33]. Consequently, IL-1 $\beta$ is has a significant effect on the metabolism of cells and the extracellular matrix [34]. A decrease in SF IL-1ra has been documented in chronic stages of human OA [35]. Delayed increase of SF IL-1ra (at day 35) following the IA administration of autologous conditioned serum in experimentally induced equine OA has been demonstrated, suggesting endogenous production of IL-1ra [2]. In the present study, IA HA had no apparent effect on SF IL-1ra concentration in the short-term. However, long-term effects of IA HA on SF IL-1ra warrants further research.

PDGF is secreted in the early inflammatory phase primarily by platelets, but also by macrophages, endothelial cells and fibroblasts [36]. It is one of the earliest and the most sensible growth factors expressed after tissue injury [37]. PDGF induces the synthesis of other growth factors [38], proliferation and differentiation of fibroblasts, deposition of collagen and angiogenesis [39, 40]. Therefore, it is an essential promoter of the healing process. In the present study, after sampling at the baseline on day 0 , the next sampling was performed on day 5 , in a time point where PDGF-BB is supposed to play a major role in the vascular formation and proliferation of fibroblasts in ongoing repair [41]. PDGF-BB concentration increased in $\mathrm{SF}$ after induction of $\mathrm{CD}$, although a significant difference was not detected. The synthesis of PDGF-BB may have, however, occurred earlier in the course of injury and inflammation. In contrast, PDGF-BB has not been detected at all in the SF of osteoarthritic human knee joint [42]. In another study comparing OA joints to healthy controls, significant differences in the SF PDGF$\mathrm{BB}$ concentrations were not detected [43]. These results are also suggestive for an early increase of SF PDGF$\mathrm{BB}$ concentration and its role in the initial phase of the pathogenesis of OA. SF PDGF concentration has been documented to increase after IA platelet rich plasmatreatment [23]. In the present study, no changes in the PDGF-BB concentrations were documented 9 days after IA HA treatment.

SF TGF- $\beta_{1}$ has been studied in vivo in normal joints and joints with osteochondrosis in foals [22] and after LPS challenge in adult horses [21]. In the present study, neither the creation of $\mathrm{CD}$ or IA HA injection caused an increase in the SF TGF- $\beta_{1}$ concentration. In contrast, TGF- $\beta_{1}$ concentration decreased following the creation of $\mathrm{CD}$, although no statistically significant difference between CD and SO joints was found (Fig. 2). This is different compared with the previous study on equine joints that reported increase of SF TGF- $\beta_{1}$ concentration following a challenge with LPS [21]. This may be explained by the more intense inflammation induced by LPS, compared with inflammation induced by $\mathrm{CD}$.

Ríos et al. [30] has shown an increase in TGF- $\beta_{1}$ concentration after LPS challenge in cartilage inflammation created in vitro. The effect was suggested to result from a possible anti-inflammatory mechanism or by direct damage of LPS to the cartilage. A similar effect has been seen after LPS challenge of synovial membrane explants in vitro [31]. Our result is more consistent with the study 

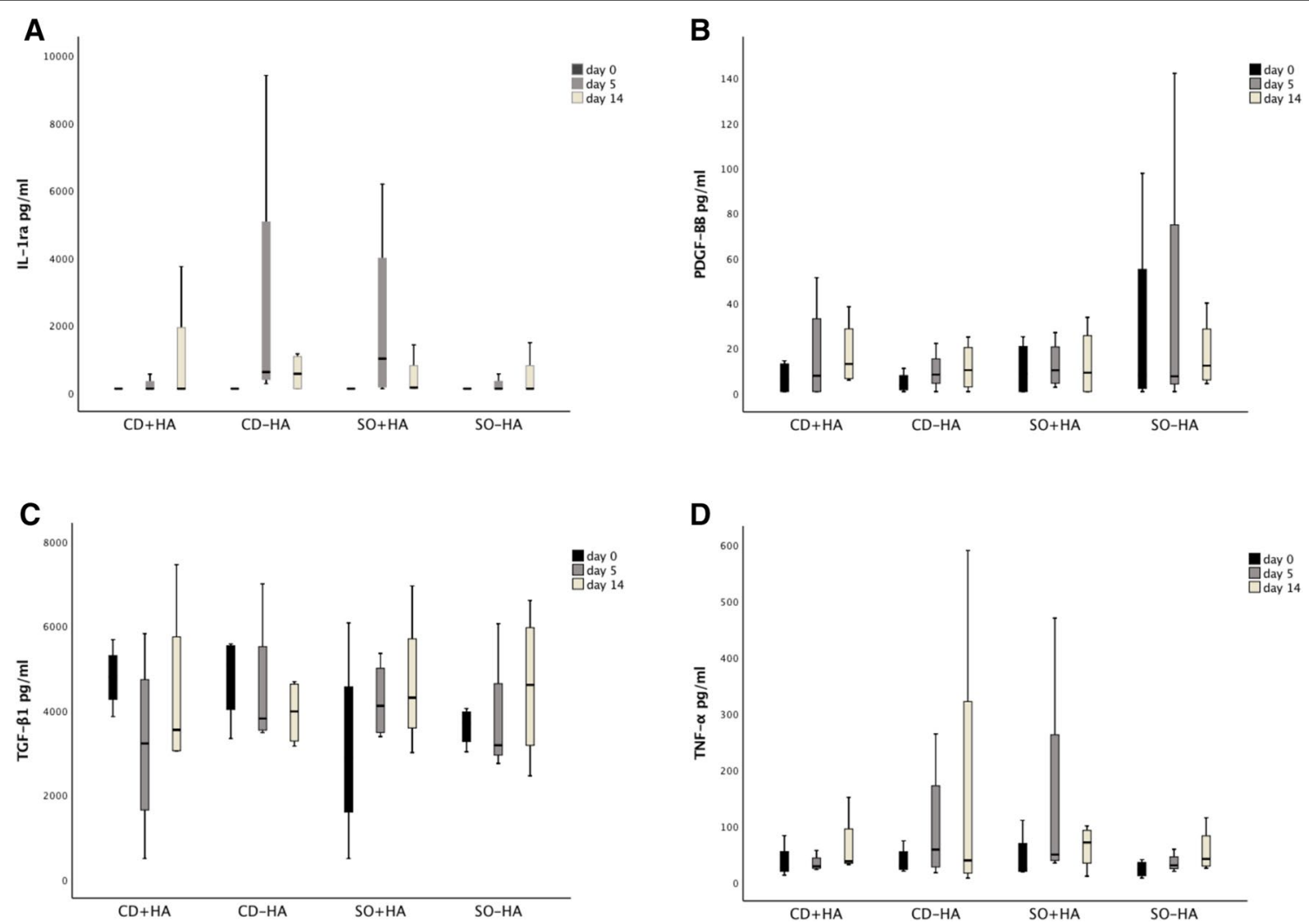

Fig. 2 SF biomarker concentrations (median, quartiles and minimum and maximum value) of treatment groups on different sampling days. $C D+H A$ cartilage defect joints with hyaluronan injection, $C D-H A$ cartilage defect joints without hyaluronan injection, $\mathrm{SO}+\mathrm{HA}$ sham-operated joints with hyaluronan injection, $\mathrm{SO}-\mathrm{HA}$ sham-operated joints without hyaluronan injection

of human OA where SF TGF- $\beta$ concentration was measured both in healthy subjects and in patients with OA; in the latter, the concentration of SF TGF- $\beta$ was low or even undetectable [44]. Cell signaling pathways may be intercepted by inflammatory cytokines, which may be a possible reason for the reduced amount of TGF- $\beta$ in the course of OA [45].

TGF- $\beta_{1}$ has an anabolic effect on cartilage; it has an ability to induce chondrogenic differentiation of mesenchymal stem cells [46, 47] with rapid biosynthesis of glycosaminoglycan and deposition of an extracellular matrix [47]. On the other hand, enhanced expression of TGF- $\beta_{1}$ has been associated with developing osteophytes [48] and hyperplasia of the synovium [49]. It has been suggested that only a narrow range of bioactive TGF- $\beta$ concentrations are beneficial to cartilage health and any concentrations below or above this range may cause aberrant alterations in TGF- $\beta$ pathways, resulting in abnormal cartilage function [50]. As TGF- $\beta$ is stored in the platelets, SF TGF- $\beta$ concentration increases after IA administration of platelet rich plasma, in response to platelet activation [23]. HA has been suggested to play an important role in the mechanical activation of latent
TGF- $\beta$ in the joint [51]. However, in the present study, no changes in the SF TGF- $\beta_{1}$ concentrations were documented after IA HA treatment, possibly implying that optimal SF TGF- $\beta_{1}$ concentrations were already present in the injected joints.

The results of studies on the synovial fluid TNF- $\alpha$ are somewhat contradictory. However, it seems to be quite a sensitive but not very specific marker of IA insults. The results of this study are also suggestive of that. TNF- $\alpha$ concentration increased in SF after induction of $C D$ although significant differences were not detected between the groups in the small population of this experimental study. An increased concentration of SF TNF- $\alpha$ has been documented in horses in naturally occurring OA in carpi [27], and experimentally in amphotericin B- [5] and LPS-induced articular inflammation [21, 26]. In horses, even exercise alone leads to a significant increase in TNF- $\alpha$ levels for a short period $[25,28]$. However, an increase in SF TNF- $\alpha$ concentration as a result from serial arthrocentesis could not be demonstrated [25]. In contrast to this, gas and liquid capsular distension during arthroscopy provoked an inflammatory response with increased concentration of 
SF TNF- $\alpha$ [52]. In a clinical equine study, TNF- $\alpha$ was found not to be a useful biomarker for different types of joint lesions [53]. Similarly, in humans, increased concentrations were not associated with any particular type of articular disease, such as rheumatoid arthritis, although in OA patients, detectable concentrations of TNF- $\alpha$ were related with a long duration of the disease [24]. In the present study, SF TNF- $\alpha$ concentrations were not affected by IA HA. Probably with more horses, a bigger sample size and earlier and more frequent sampling significant results may have been demonstrated in this study.

In conclusion, after a mild increase in biomarker concentrations resulting from arthroscopy and induction of synovitis and CD, HA failed to produce any further effect on biomarkers. Minor improvement of clinical signs of IA inflammation was evident in the $\mathrm{CD}+\mathrm{HA}$ group, when compared with scores of clinical signs of $\mathrm{CD}-\mathrm{HA}$ group. However, in $\mathrm{SO}+\mathrm{HA}$ and $\mathrm{SO}-\mathrm{HA}$ groups changes after IA HA/no injection were not that consistent. HA is reported to have an anti-inflammatory effect [16] but IA HA injections have also been documented to induce a transitional IA inflammatory reaction, either a flare with pronounced clinical signs of inflammation and pain [54] or an increase of WBC count in the SF [11]. To our knowledge, only a few studies have explored biomarkers in equine SF after IA HA $[3,11]$. As HA is very frequently used in the IA treatment in horses, its mechanism of action warrants further research.

This study has its limitations. Firstly, the contralateral limb of the horse served as a SO control. Concentrations of cartilage matrix products are elevated also in the contralateral knee in patients with anterior cruciate ligament rupture, possibly as a consequence of an altered loading [55]. Cytokines and degraded matrix products released from an operated joint may be transported to the contralateral joint by the circulation and initiate an inflammatory process. Therefore, the concentration of markers in the control joints may have resulted partly by the transport from the CD operated joint. Measurement of serum concentrations of studied markers, as well as additional pro-inflammatory markers and markers of cartilage matrix metabolism, would have provided additional information to test this hypothesis. However, this was outside the scope of this study. Moreover, the arthroscopy itself, as well as harvesting the synovial tissue samples, causes trauma to the synovial soft tissues. These may have further affected the concentrations of the measured markers in the SO joints. However, the effect was thought to be transient. To minimize the effect of arthroscopy and sampling of synovial soft tissue, sampling of SF and IA HA injection were performed only after 5 days.
The number of horses in the experimental studies on equine joints has generally ranged from 6 to $13[1,3,5$, 21, 26, 52]. Eight horses (16 joints) in the present study may have been too low to detect differences in concentrations of SF biomarkers. Although horses with uniform breed and sport discipline were selected, and all horses were free of lameness and joint disease of the intercarpal joint as verified by arthroscopy, differences in exercise or training status and age may have caused some variation in the SF biomarker concentrations. Exercise may lead to increased biomarker concentrations in horses [7, 28 , which can be further enhanced in joints with compromised health [7, 25]. Moreover, also age has been reported to influence SF biomarker concentrations or gene expression so that they generally decrease with age $[8,29,56]$.

The time from injury to collection of SF samples is an important issue as changes in biomarker concentrations can occur quickly, even within hours. On the other hand, IA injection itself, especially repeatedly, causes inflammatory reaction in the joint [57] and can cause increases in biomarker concentrations [56]. To minimize this effect, it has been recommended there should be a period of even 14 days after the previous arthrocentesis before subsequent SF collection [28]. However, despite of these potentially interfering factors, repeated sampling and early time points have been generally used in equine SF biomarker studies. In studies using LPS induction for IA inflammation, first sampling point $8 \mathrm{~h}$ [19] or $1 \mathrm{~h}$ $[21,26]$ post injection were chosen which is reasonable considering very acute and strong inflammation caused by LPS. On the other hand, in some experimental studies, using surgical model $[3,4]$ comparable with model of the present study or amphotericin B [5] for induction of IA inflammation in horses, weekly samplings were chosen for exploring SF IL-1ra and TNF- $\alpha$ concentrations (among other biomarkers), respectively. Finding an optimal time point for SF aspiration is challenging, especially when several biomarkers are studied. In addition, avoiding the effect of repeated aspiration and sham-operation on biomarker concentrations complicates the issue. Although the effect of IA injection on biomarkers measured in this study is not known, except for TNF- $\alpha, 5$ days after arthroscopy and 9 days after IA HA injection were chosen to balance between the intervention time points.

\section{Conclusions}

This study demonstrates that arthroscopy and both the creation of CD and SO, lead to an increase in SF IL-1ra and TNF- $\alpha$ concentrations but changes in concentrations of anabolic growth factors TGF- $\beta_{1}$ and PDGF-BB in SF were not documented 5 days after the arthroscopy. Intraarticular HA was well tolerated. However, changes in 
concentrations of IL-1ra, PDGF-BB, TGF- $\beta$, and TNF- $\alpha$ were not seen after HA injection. The treatment effect of IA HA on SF pro-inflammatory, anti-inflammatory and anabolic biomarkers warrants further research.

\begin{abstract}
Abbreviations
CD: cartilage defect; EDTA: etylenediaminetetraacetic acid; HA: hyaluronan; IA: intra-articular; IL-1 ra: interleukin 1 receptor antagonist; LPS: lipopolysaccharide; NASHA: non-animal stabilized hyaluronic acid; OA: osteoarthritis; PDGF-BB: platelet-derived growth factor; $\mathrm{PGE}_{2}$ : prostaglandin $\mathrm{E}_{2} ; \mathrm{SF}$ : synovial fluid; $\mathrm{SO}$ : sham-operation; TGF- $\beta_{1}$ : transforming growth factor beta 1; TNF-a: tumour necrosis factor alpha; WBC: white blood cell.
\end{abstract}

\section{Acknowledgements}

Financial support from the Finnish Foundation of Veterinary Research, the Finnish Veterinary Foundation, and the Erkki Rajakoski Foundation of the Finnish Trotting Association is acknowledged. The authors thank Teppo Huttunen for statistical assistance.

\section{Authors' contributions}

TMN performed the clinical study and arthroscopy of all horses, evaluation of radiographs, and drafted the manuscript. CL and JUC contributed to the laboratory work, statistical analyses, interpretation of results and revision of the manuscript. R-MT participated in the design of the study, interpretation of results and revision of the manuscript. All the authors read and approved the final manuscript.

\section{Funding}

The study was supported in part by a grant from the Finnish Foundation of Veterinary Research, the Finnish Veterinary Foundation, and the Erkki Rajakoski Foundation of the Finnish Trotting Association, which did not have any role in the design, collection, analysis, or interpretation of the data or in writing the manuscript.

\section{Availability of data and materials}

The datasets analysed during the current study are available from the corresponding author on reasonable request.

\section{Ethics approval and consent to participate}

The study protocol was approved by the National Animal Experimentation Board of Finland (ESAVI/1130/04.10.03/2011).

\section{Consent for publication}

Not applicable.

\section{Competing interests}

The authors declare that they have no competing interests.

\section{Author details}

1 Department of Equine and Small Animal Medicine, Faculty of Veterinary Medicine, University of Helsinki, P.O. Box 57, 00014 Helsinki, Finland. ${ }^{2}$ Grupo de Investigación Terapia Regenerativa, Departamento de Salud Animal, Universidad de Caldas, Calle 65 No26-10, Manizales, Caldas, Colombia.

Received: 29 December 2018 Accepted: 27 May 2019

Published online: 30 May 2019

\section{References}

1. Frisbie DD, Ghivizzani SC, Robbins PD, Evans CH, Mcllwraith CW. Treatment of experimental equine osteoarthritis by in vivo delivery of the equine interleukin-1 receptor antagonist gene. Gen Ther. 2002;9:12-20.

2. Frisbie DD, Kawcak CE, Werpy NM, Park RD, Mcllwraith CW. Clinical, biochemical, and histologic effects of intra-articular administration of autologous conditioned serum in horses with experimentally induced osteoarthritis. Am J Vet Res. 2007:68:290-6.
3. Frisbie DD, Kawcak CE, Mcllwraith CW, Werpy NM. Evaluation of polysulfated glycosaminoglycan or sodium hyaluronan administered intra-articularly for treatment of horses with experimentally induced osteoarthritis. Am J Vet Res. 2009;70:203-9.

4. Frisbie DD, Mcllwraith CW, Kawcak CE, Werpy NM. Evaluation of intraarticular hyaluronan, sodium chondroitin sulfate and $\mathrm{N}$-acetyl-D-glucosamine combination versus saline $(0.9 \% \mathrm{NaCl})$ for osteoarthritis using an equine model. Vet J. 2013;197:824-9.

5. Ma TW, Li Y, Wang GY, Xin-ran Li XR, Jiang RL, Song XP, et al. Changes in synovial fluid biomarkers after experimental equine osteoarthritis. J Vet Res. 2017;61(10):1515.

6. Bertone A, Palmer JL, Jones J. Synovial fluid cytokines and eicosanoids as markers of joint disease in horses. Vet Surg. 2001;30:528-38.

7. Frisbie DD, Al-Sobayil F, Billinghurst RC, Kawcak CE, Mcllwraith CW. Changes in synovial fluid serum biomarkers with exercise and early osteoarthritis in horses. Osteoarthr Cartilage. 2008;16:1197-204.

8. Nicholson AM, Trumble TN, Merritt KA, Brown MP. Association of horse age, joint type, and osteochondral injury with serum and synovial fluid concentrations of type II collagen biomarkers in Thoroughbreds. Am J Vet Res. 2010;71:741-9.

9. Wojdadiewicz LA, Poniatowski D, Szukiewicz D. The role of inflammatory and anti-inflammatory cytokines in the pathogenesis of osteoarthritis. Mediators Inflamm. 2014. https://doi.org/10.1155/2014/561459.

10. Goodrich $L R$, Nixon AJ. Medical treatment of osteoarthritis in the horsea review. Vet J. 2006;171:51-69.

11. Niemelä TM, Tulamo R-M, Aaltonen K, Sankari SM, Hielm-Björkman AK. Change in biomarkers in equine synovial fluid two weeks after intraarticular hyaluronan treatment: a randomized double-blind clinical trial. BMC Vet Res. 2018;14:186.

12. Johansen M, Bahrt H, Altman RD, Bartels EM, Juhl CB, Bliddal H, et al. Exploring reasons for the observed inconsistent trial reports on intraarticular injections with hyaluronic acid in the treatment of osteoarthritis: meta-regression analyses of randomized trials. Semin Arthritis Rheum. 2016;46:34-48.

13. Punzi L, Sciavon F, Cavasin F, Ramonda R, Gambari PF, Todesco S. The influence of intra-articular hyaluronic acid on $\mathrm{PGE}_{2}$ and $\mathrm{CAMP}$ of synovial fluid. Clin Exp Rheumatol. 1989;7:247-50.

14. Chang NS, Intrieri C, Mattison J, Armand G. Synthetic polysulfated hyaluronic acid is a potent inhibitor for tumour necrosis factor production. J Leucocyte Biol. 1994;55:778-84.

15. Cole BJ, Karas V, Hussey K, Pilz K, Fortier LA. Hyaluronic acid versus platelet-rich plasma: a prospective, double-blind randomized controlled trial comparing clinical outcomes and effects on intra-articular biology for the treatment of knee osteoarthritis. Am J Sports Med. 2017;45:339-46.

16. Gomis A, Pawlak M, Balazs EA, Schmidt RF, Belmonte C. Effects of different molecular weight elastoviscous hyaluronan solutions on articular nociceptive afferents. Arthritis Rheum. 2004;50:314-26.

17. Caires R, Luis E, Taberner FJ, Fernandez-Ballester G, Ferrer-Montiel A, Balazs EA, et al. Hyaluronan modulates TRPV1 channel opening, reducing peripheral nociceptor activity and pain. Nat Commun. 2015;27:6.

18. Anonymous. Definition and Classification of Lameness. In: Guide for Veterinary Service and Judging of Equestrian Events. In: Lexington, American Association of Equine Practitioners. 1991, pp. 19.

19. de Grauw JC, van de Lest CHA, Brama PAJ, Rambags BPB, van Weeren PR. In vivo effects of meloxicam on inflammatory mediators, MMP activity and cartilage biomarkers in equine joints with acute synovitis. Equine Vet J. 2009:41:693-9.

20. Ríos DL, López C, Carmona JU. Platelet-rich gel supernatants stimulate the release of anti-inflammatory proteins on culture media of normal equine synovial membrane explants. Vet Med Int. 2015. https://doi. org/10.1155/2015/547052.

21. Carmalt UL, Bell CD, Tatarniuk DM, Suri SS, Singh B, Waldner C. Comparison of the response to experimentally induced short-term inflammation in the temporomandibular and metacarpophalangeal joints of horses. Am J Vet Res. 2011;72:1586-91.

22. Grauw JC, Donabédian M, Lest CHA, Perona G, Robert C, Lepage O, et al. Assessment of synovial fluid biomarkers in healthy foals and in foals with tarsocrural osteochondrosis. Vet J. 2011:190:390-5.

23. Textor JA, Willits NH, Tablin F. Synovial fluid growth factor and cytokine concentrations after intra-articular injection of a platelet-rich product in horses. Vet J. 2013:198:217-23. 
24. di Giovine FS, Nuki G, Duff GW. Tumour necrosis factor in synovial exudates. Ann Rheum Dis. 1988;47:768-72.

25. Billinghurst RC, Fretz PB, Gordon JR. Induction of intra-articular tumour necrosis factor during acute inflammatory responses in equine arthritis. Equine Vet J. 1995;27:208-16.

26. Hawkins DL, Cargile JL, MacKay RJ, Broome TA, Skelley LA. Effect of tumor necrosis factor antibody on synovial fluid cytokine activities in equine antebrachiocarpal joints injected with endotoxin. Am J Vet Res. 1995:56:1292-9.

27. Kamm JL, Nixon AJ, Witte TH. Cytokine and catabolic enzyme expression in synovium, synovial fluid and articular cartilage of naturally osteoarthritic equine carpi. Equine Vet J. 2010:42:693-9.

28. van den Boom R, Brama PAJ, Kiers GH, DeGroot J, Barneveld A, van Weeren PR. The influence of repeated arthrocentesis and exercise on matrix metalloproteinase and tumor necrosis factor a activities in normal equine joints. Equine Vet J. 2004;36:155-9.

29. Iqpal J, Dudhia J, Bird JLE, Bayliss MT. Age-related effects of TGF-b on proteoglycan synthesis in equine articular cartilage. Biochem Bioph Res Co. 2000;274:467-71.

30. Ríos DL, López C, Carmona JU. Evaluation of the anti-inflammatory effects of two platelet-rich gel supernatants in an in vitro system cartilage inflammation. Cytokine. 2015;76:505-13.

31. Ríos DL, López C, Carmona JU. Effects over time of two platelet gel supernatants on growth factor, cytokine and hyaluronan concentrations in normal synovial membrane explants challenged with lipopolysaccharide. BMC Musculoskelet Disord. 2015;16:153.

32. Haller JM, McFadden M, Kubiak EN, Higgins TF. Inflammatory cytokine response following acute tibial plateau fracture. J Bone Joint Surg Am. 2015;97:478-83.

33. Roman-Blas JA, Jimenez SA. NF-KB as a potential therapeutic target in osteoarthritis and rheumatoid arthritis. Osteoarthr Cartilage. 2006;14:839-48.

34. Marcu KB, Otero M, Olivotto E, Borzi RM, Goldring MB. NF-KB signalling: multiple angles to target OA. Curr Drug Targets. 2010;11:599-613.

35. Bigoni M, Turati M, Sacerdote P, Gaddi D, Piatti M, Castelnuovo A, et al. Characterization of synovial fluid cytokine profiles in chronic meniscal tear of the knee. J Orthop Res. 2017;35:340-6.

36. Seppä H, Grotendorst G, Seppä S, Schiffmann E, Martin GR. Plateletderived growth factor is chemotactic for fibroblasts. J Cell Biol. 1982;92:584-8.

37. Girolamo L, Galliera E, Volpi P, Denti M, Dogliotti G, Quaglia A, et al. Why menisci show higher healing rate when repaired during $A C L$ reconstruction? Growth factors release can be the explanation. Knee Surg Sports Traumatol Arthrosc. 2015;23:90-6.

38. Creaney L, Hamilton B. Growth factor delivery methods in the management of sports injuries: the state of play. Br J Sports Med. 2008;42:314-20.

39. Mustoe TA, Pierce GF, Morishima C, Deuel TF. Growth factor-induced acceleration of tissue repair through direct and inductive activities in a rabbit dermal ulcer model. J Clin Invest. 1991;87:694-703.

40. Wu L, Brucker M, Gruskin E, Roth SI, Mustoe TA. Differential effects of platelet-derived growth factor BB in accelerating wound healing in aged versus young animals: the impact of tissue hypoxia. Plast Reconstr Surg. 1997:99:815-22.

41. Anitua E, Sanchez M, Zalduendo MM, de la Fuente M, Prado R, Orive G, et al. Fibroblastic response to treatment with different preparations rich in growth factors. Cell Prolif. 2009;42:162-70.

42. Mabey T, Honsawek S, Saetan N, Poovorawan Y, Tanavalee A, Yuktanandana P. Angiogenic cytokine expression profiles in plasma and synovial fluid of primary knee osteoarthritis. Int Orthop. 2014;38:1885-92.
43. Beekhuizen M, Gierman LM, van Spil WE, Van Osch GJVM, Huizinga TWJ, Saris DBF, et al. An explorative study comparing levels of soluble mediators in control and osteoarthritic synovial fluid. Osteoarthr Cartilage. 2013;21:918-22.

44. Blaney Davidson EN, Vitters EL, Van Der Kraan PM, Van Den Berg WB. Expression of transforming growth factor- $\beta$ (TGF $\beta$ ) and the TGF $\beta$ signaling molecule SMAD-2P in spontaneous and instability-induced osteoarthritis: role in cartilage degradation, chondrogenesis and osteophyte formation. Ann Rheum Dis. 2006;65:1414-21.

45. Derynck R, Zhang YE. Smad-dependent and Smad-independent pathways in TGF- $\beta$ family signaling. Nature. 2003;425:577-84.

46. Barry F, Boynton RE, Liu B, Murphy JM. Chondrogenic differentiation of mesenchymal stem cells from bone marrow: differentiation-dependent gene expression of matrix components. Exp Cell Res. 2001;268:189-200.

47. Tuli R, Tuli S, Nandi S, Huang X, Manner PA, Hozack WJ, et al. Transforming growth factor- $\beta$-mediated chondrogenesis of human mesenchymal progenitor cells involves $\mathrm{N}$-cadherin and mitogen activated protein kinase and Wnt signaling cross-talk. J Biol Chem. 2003;278:41227-36.

48. Scharstuhl A, Glansbeek HL, van Beuningen HM, Vitters EL, van der Kraan PM, van den Berg WB. Inhibition of endogenous TGF-beta during experimental osteoarthritis prevents osteophyte formation and impairs cartilage repair. J Immunol. 2002;169:507-14.

49. Backer AC, van de Loo FA, van Beuningen HM, Sime $P$, van Lent PL, van der Kraan PM, et al. Overexpression of active TGF-beta-1 in the murine knee joint: evidence for synovial-layer-dependent chondro-osteophyte formation. Osteoarthr Cartilage. 2001;9:128-36.

50. Finnson KW, Chi Y, Bou-Gharios G, Leask A, Philip A. TGF-b signaling in cartilage homeostasis and osteoarthritis. Front Biosci (Schol Ed). 2012;4:251-68.

51. Albro MB, Cigan AD, Nims RJ, Yerishalmi KJ, Oungoulian SR, Hung CT, et al. Shearing of synovial fluid activates latent TGF- $\beta$. Osteoarthr Cartilage. 2012;20:1374-82.

52. Rossetti RB, de Oliveira Massoco C, Penna ACA, da Silva LCL. An experimental study to compare inflammatory response due to liquid or gas joint distension in horses submitted to arthroscopy. Acta Cir Bras. 2012;72:848-54.

53. Ley C, Ekman S, Elmén A, Nilsson G, Eloranta M-L. Interleukin-6 and tumor necrosis factor in synovial fluid from horses with carpal joint pathology. Equine Vet J. 2004;36:155-9.

54. Marino AA, Waddell DD, Kolomytkin OV, Pruett S, Sadasivan S, Kalia K, et al. Assessment of immunological mechanism for flare reactions to Synvisc ${ }^{\circledR}$. Clin Orthop Relat Res. 2006;442:187-94.

55. Dahlberg L, Roos H, Saxne T, Heinegard D, Lark MW, Hoerrner LA, et al. Cartilage metabolism in the injured and uninjured knee of the same patient. Ann Rheum Dis. 1994;53:823-7.

56. Brama PA, van den Boom R, DeGroot J, Kiers GH, van Weeren PR. Collagenase-1 (MMP-1) activity in equine synovial fluid: influence of age, joint pathology, exercise and repeated arthrocentesis. Equine Vet J. 2004;36:34-40.

57. Tulamo R-M, Bramlage LR, Gabel AA. Sequential clinical and synovial fluid changes associated with acute infectious arthritis in the horse. Equine Vet J. 1989;5:325-31.

\section{Publisher's Note}

Springer Nature remains neutral with regard to jurisdictional claims in published maps and institutional affiliations. 